\title{
Visual impairment in immunoglobulin G4-related disease: A case report
}

\author{
XUE WANG ${ }^{*}$, XUREN SUN ${ }^{*}$, RAN AO, ZILU ZENG, DAN LI and YILING LI \\ Department of Gastroenterology, The First Affiliated Hospital of China Medical University, \\ Shenyang, Liaoning 110000, P.R. China
}

Received September 8, 2019; Accepted December 8, 2020

DOI: 10.3892/etm.2021.9633

\begin{abstract}
The present study reports on the case of a 50-year-old male with sinusitis, diplopia, secretory otitis media and skin eczema for $>14$ years. The patient presented with visual impairment in both eyes and subxiphoid pain on admission to the First Affiliated Hospital of China Medical University (Shenyang, China). Orbital CT revealed a slightly thickened left inferior rectus muscle. Due to a periocular mass, enlarged lymph nodes, elevated serum immunoglobulin G4 (IgG4) levels and enriched IgG4-positive plasma cells in the lymph nodes, the diagnosis of IgG4-related disease (IgG4-RD), possibly involving at least 10 organs, was established. Following treatment with methylprednisolone, the serum IgG4 levels decreased to normal and binocular vision returned to normal. Unlike previously reported cases, the present case exhibited no swollen masses around the optic nerve. The purpose of the present case report was to improve the understanding of IgG4-RD.
\end{abstract}

\section{Introduction}

Immunoglobulin G4-related disease (IgG4-RD) is a regional or systemic fibroinflammatory disease of unknown etiology (1) that predominantly occurs in middle-aged and elderly males (40-70 years old). It is characterized by compressive lesions with inflammation or neoplastic swelling, usually but not always elevated serum IgG4 levels and a typical histopathological appearance. The key pathological features are a dense

Correspondence to: Ms Dan Li or Dr Yiling Li, Department of Gastroenterology, The First Affiliated Hospital of China Medical University, 155 Nanjing North Street, Heping, Shenyang, Liaoning 110000, P.R. China

E-mail: 285981349@qq.com

E-mail: lyl-72@163.com

${ }^{*}$ Contributed equally

Key words: visual impairment, immunoglobulin G4-related disease, immunoglobulin G4-related ophthalmopathy, steroid therapy, inflammatory pseudotumor, Graves' ophthalmopathy lymphoplasmacytic infiltrate, particularly IgG4-positive cells, a storiform pattern of fibrosis and obliterative phlebitis $(1,2)$. The disease may involve the eyes, salivary glands, lymph nodes, pituitary glands, dura mater, thyroid, lung, pleura, breast, pericardium, aorta, retroperitoneum, pancreas, bile duct, kidney, prostate and skin.

It was reported that the prevalence of autoimmune pancreatitis (AIP) was 10.1/100,000 individuals in Japan in 2016 (3). When IgG4-positive cells infiltrate the lacrimal glands, orbital soft tissues or extraocular muscles, it is referred to as IgG4-related ophthalmopathy (IgG4-ROD) (4). Due to the rare involvement of the optic nerve, visual impairment is an uncommon symptom. The present study reported on the case of a patient diagnosed with IgG4-RD probably involving at least 10 organs with visual impairment as the major complaint, who exhibited sensitivity to hormone therapy with obvious therapeutic effects.

\section{Case report}

A 50-year-old male presented at the First Affiliated Hospital of China Medical University (Shenyang, China) with repeated diplopia for 14 years, visual impairment for 2 months and subxiphoid pain for 1 month in May 2019. The patient had suffered from periocular swelling and diplopia repeatedly since 2005, but he did not complain of visual impairment until nearly two months prior to admission. The patient was treated several times for the above symptoms. He was diagnosed with an inflammatory pseudotumor and then subjected to pseudotumor resection and postoperative radiotherapy. The patient had also been diagnosed with Graves' ophthalmopathy with normal thyroid function and given hormone shock therapy numerous times. However, the prognosis of the patient was unsatisfactory, and the symptoms kept on recurring. At one month prior to admission, the patient developed subxiphoid pain lasting $\sim 3 \mathrm{~h}$ after eating yogurt, accompanied by nausea and vomiting. The symptoms occurred repeatedly thereafter, more frequently after eating. The pain lasted $\sim 2-3$ min and improved by itself. The patient also had problems with urinary weakness. The patient exhibited a reduction in body weight by $5 \mathrm{~kg}$ over the past 2 months. He had a history of sinusitis for 30 years, secretory otitis media for 7 years and skin eczema for 7 years. An endoscopic operation was performed for sinusitis and secretory otitis media in 2013. 
Physical examination revealed the following: Multiple enlarged lymph nodes in the left mandible, bilateral neck and bilateral supraclavicular fossa were palpated; these nodes were of medium hardness, movable and painless. Bilateral eyelid edema and a left lower eyelid mass were discovered.

Laboratory investigation revealed the following: All enzymes representing liver and bile duct damage were elevated, including alanine aminotransferase (ALT) at $160 \mathrm{U} / 1$ (normal range, 9-50 U/l), aspartate aminotransferase (AST) at $142 \mathrm{U} / 1$ (normal range, 15-40 U/1), $\gamma$-glutamyl transpeptidase (GGT) at $742 \mathrm{U} / 1$ (normal range, 10-60 U/1) and alkaline phosphatase (ALP) at $467 \mathrm{U} / 1$ (normal range, 45-125 U/1). Direct bilirubin was slightly elevated to $8.3 \mu \mathrm{mol} / 1$ (normal range, 0-6.8 $\mu \mathrm{mol} / \mathrm{l}$ ), while albumin was severely reduced to $21.2 \mathrm{~g} / 1$ (normal range, 40-55 g/1). Both amylase (AMY) and lipase (LPS) were increased to $120 \mathrm{U} / 1$ (normal range, 28-100 U/1) and 136.1 U/1 (normal range, 13-60 U/l), respectively. The erythrocyte sedimentation rate and procalcitonin were also increased. The T cell spot test was positive. Except for elevated thyroid stimulating hormone (TSH) and anti-thyroglobulin antibody, there were no thyroid function abnormalities [free thyroid (FT) hormone 3, FT4 and anti-thyroid peroxidase antibody were all normal]. Both $\mathrm{IgG}$ and $\mathrm{IgG} 4$ were elevated to $85.44 \mathrm{~g} / 1$ (normal range, $7-17 \mathrm{~g} / \mathrm{l}$ ) and $36.9 \mathrm{~g} / 1$ (normal range, 0.03-2.01 g/l), respectively. Antinuclear antibody was positive. The antigen spectrum of autoimmune liver disease (e.g., anti-mitochondrial antibody-M2), anti-hepatorenal microsomes-1, anti-hepatocyte cytoplasmic antigen type 1 antibody, anti-soluble liver antigen/hepatopancreas antigen antibody, Ro-52, anti-promyelocytic leukemia protein antibody, nuclear autoantigen Sp-100, nuclear pore protein gp210 and 2-ketoacid dehydrogenase complex was negative. Other markers, such as C-reactive protein, complement, immunofixation electrophoresis, coagulation, hepatitis, viral antibody and tumor markers, were normal. The right intraocular pressure was $14 \mathrm{mmHg}$ and the left intraocular pressure was $16 \mathrm{mmHg}$. The visual acuity of both eyes was 0.6. Defects in the right lower half, right upper half and left infratemporal region were detected in the 30-degree visual field. The synoptic muscle exhibited left inferior oblique paralysis. Orbital CT (Fig. 1) revealed a slightly thickened left inferior rectus muscle and paranasal sinusitis. Mild interstitial changes, slight chronic inflammation in both lungs, small nodules in both lungs, and mediastinal and bilateral axillary lymphadenopathy were detected on pulmonary CT. Prostate hypertrophy and calcification and lymphadenopathy around the bilateral internal iliac vessels were also observed on abdomen-enhanced CT. An ultrasound scan of the superficial lymph nodes revealed that the bilateral neck, bilateral supraclavicular fossa, bilateral subclavian fossa, submental, left axilla, right groin (grades 3-4, suspected malignancy) (Fig. 2), right axilla and left groin (grade 3 ) exhibited multiple enlarged lymph nodes. A hypoechoic mass was detected in the left submandibular gland. Rough echo of the liver parenchyma and increased elasticity of the liver (10.6 kpa) were observed in the abdominal ultrasound scan.

The patient underwent biopsy of the left inguinal lymph node. Pathological analysis indicated $>10$ IgG4-positive cells in the marginal sinus perhigh-power field (magnification, x200; Fig. 3) and scattered plasma-cell infiltration was observed per

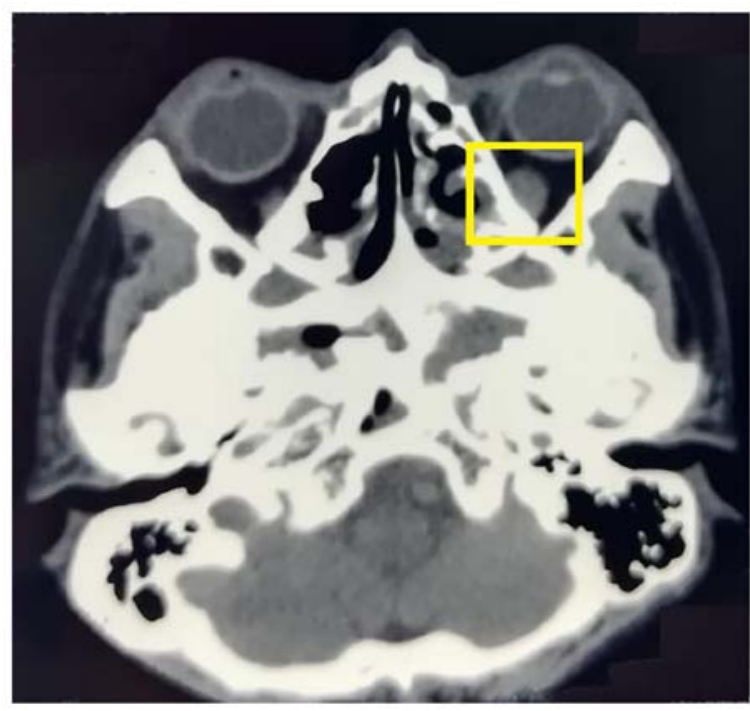

Figure 1. A thickened left inferior rectus muscle prior to treatment was observed on orbital CT (highlighted area).

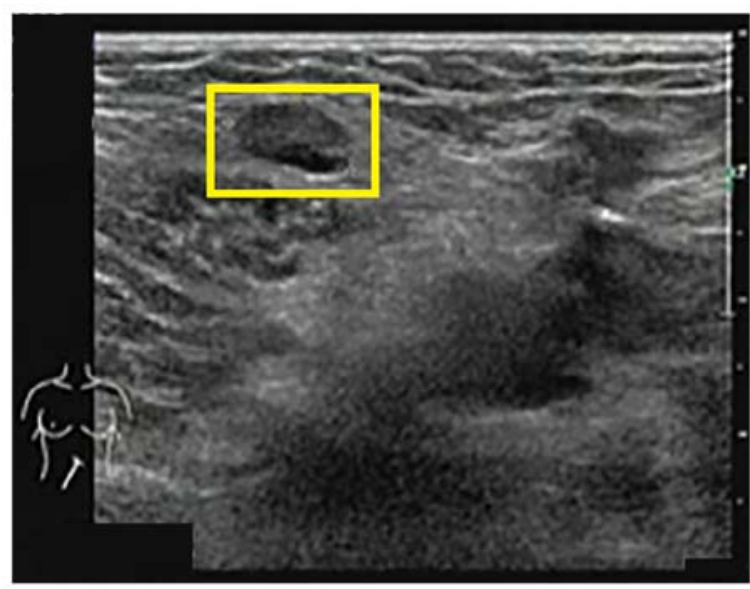

Figure 2. Ultrasound scan of the right groin displaying an enlarged lymph node (highlighted area; scale bar, $4 \mathrm{~cm}$ ).

high-power field (magnification, x400; Fig. 4). The immunohistochemistry results were as follows: Creatine kinase (-), CD3 (diffuse +), CD20 (germinal center +), CD30 (scatter +), paired box protein Pax-5 (germinal center + ), Bcl-2 (follicular-), CD21 (dendritic cells +), CD68 (scatter +), Ki-67 ( 15\%), CD138 $($ focal + ), CD38 (partial +), CD1a (scatter + ), S-100 (focal +) and IgG4 (scatter + cells could be observed in the subcapsular sinus and medullary sinus). According to the latest diagnostic criteria of the Japanese Study Group in 2011 (5), the patient was diagnosed with IgG4-RD involving the lymph nodes.

The patient's medical records from visits to other hospitals in 2013 were collected. A binocular MRI scan and enhancement indicated protruding bilateral eyeballs, bilateral intraorbital lacrimal glands and a lower left orbital mass with a slightly longer T1 signal, a slightly shorter T2 signal, poor display of lacrimal glands, displacement of corresponding horizontal extraocular muscles and eyeballs, local enlargement and irregularly shaped extraocular muscles. Following 


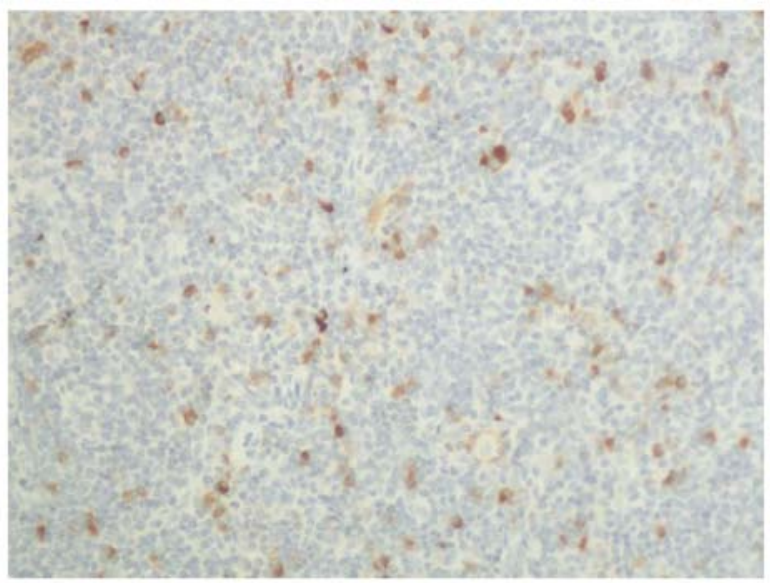

Figure 3. Pathological image of the left inguinal lymph node containing $>10$ IgG4-positive cells in the marginal sinus of the lymph node per high-power field (IgG4 staining; magnification, x200). IgG, immunoglobulin G.

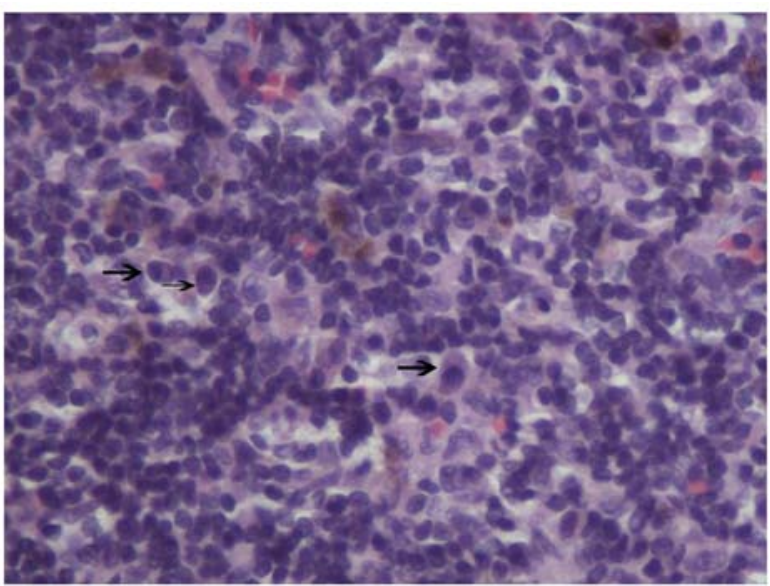

Figure 4. Scattered plasma-cell infiltration was observed in a pathology image of the left inguinal lymph node (arrows; high-power field; magnification, $\mathrm{x} 400$ ).

injection of GD-DPTA (a contrast agent), the lesions displayed with moderate contrast and homogeneous enhancement. In the pathological sections of the periocular mass, $>10 \mathrm{IgG} 4$-positive cells per high-power field were determined (magnification, x200; Fig. 5), which was consistent with the pathological features of IgG4-RD. Finally, the patient was diagnosed with IgG4-ROD. The previous misdiagnosis may have been due to an insufficient understanding of IgG4-RD.

Based on the patient's medical history and previous studies $(1,6-8)$, it was indicated that the submandibular glands, sinuses, middle ears, bile duct, liver, pancreas, prostate and skin may also be involved in IgG4-RD. IgG4-RD is most prevalent in older males (1), and in this patient population prostate problems are very common, therefore there may be a connection.

The patient was treated with $120 \mathrm{mg}$ of intravenous methylprednisolone daily, which was gradually reduced to $40 \mathrm{mg} /$ day after three weeks, and then decreased by $5 \mathrm{mg}$ every two weeks. Considering the latent infection with tuberculosis bacterium, $0.3 \mathrm{~g}$ oral isoniazid was administered once daily

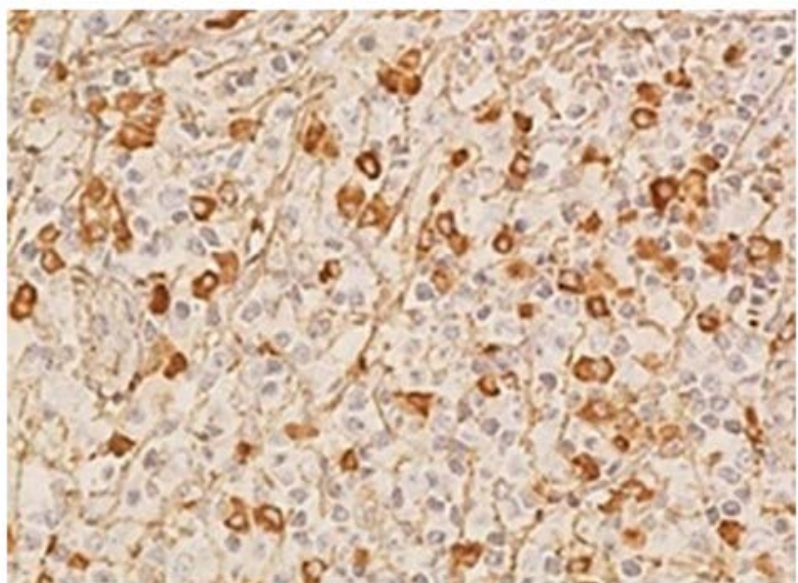

Figure 5. Pathology of the periocular mass in 2013 (before the patient was presented at the present hospital) revealed $>10 \mathrm{IgG} 4$-positive cells per high-power field (IgG4 staining; magnification, x200). $\mathrm{IgG}$, immunoglobulin $\mathrm{G}$.

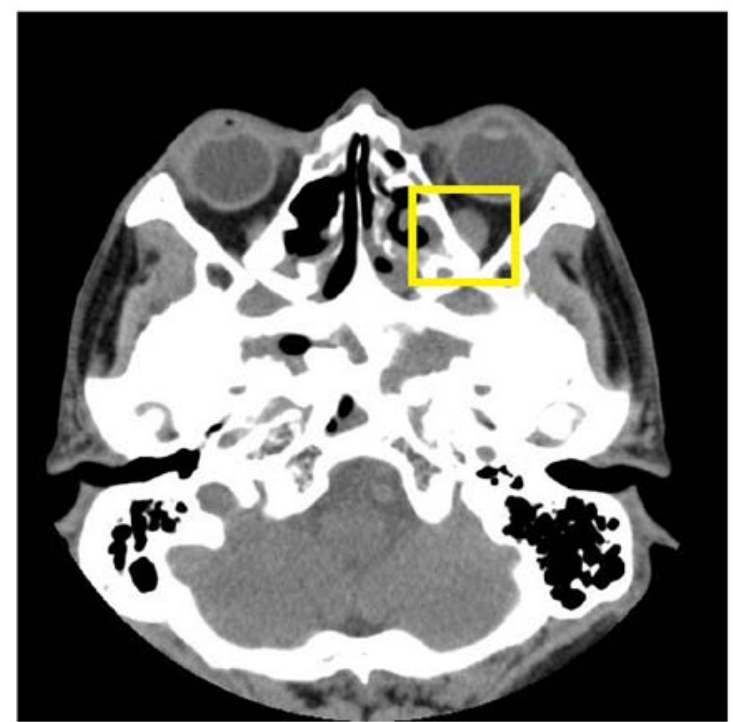

Figure 6. Slight narrowing of the enlarged left inferior rectus muscle after three months of treatment was observed on orbital CT (highlighted area).

to prevent tuberculosis. After oral administration of methylprednisolone for two months, the eyelid edema was relieved, the left lower eyelid mass became smaller and the bilateral neck and left submandibular lymph nodes were significantly reduced. The symptoms of urination and eczema, smelling caused by sinusitis and hearing caused by secretory otitis media were all improved and no nausea or vomiting symptoms occurred. ALT, AST, GGT and ALP were all decreased to $25,17,80$ and $70 \mathrm{U} / 1$, respectively. AMY and LPS became normal. IgG4 decreased to $1.32 \mathrm{~g} / \mathrm{l}$ (normal level) and albumin increased to $37 \mathrm{~g} / 1$.

The lymph nodes of the bilateral neck, bilateral supraclavicular fossa and submandibular gland became smaller on the ultrasound scan. The lymph node grade at the first two sites changed to grade 2, excluding malignancy. Orbital CT revealed that the enlarged left inferior rectus muscle became slightly narrowed (Fig. 6). The visual acuity of both eyes 
exhibited marked improvements and the vision of both eyes increased to 1.0 .

\section{Discussion}

The patient described in the present study had a long history of hospital visits with large expenses. He was misdiagnosed with an 'inflammatory pseudotumor' and 'Graves' ophthalmopathy' and the treatment effects were poor. An accurate diagnosis of IgG4-RD was difficult. The proposed IgG4-RD was based on cumulative evidence of AIP and related extrapancreatic lesions. In 2001 and 2002, Hamano et al $(9,10)$ reported an elevation in the serum IgG4 concentration in patients with AIP and the infiltration of IgG4-positive plasma cells in the pancreas and retroperitoneal tissues, respectively. In 2003, Kamisawa et al (11) reported that IgG4-positive plasma cells invaded the pancreas and extrapancreatic tissue in type 1 AIP and suggested that this phenomenon may be referred to as IgG4-RD. Ultimately, the diagnostic criteria for IgG4-RD were established in 2011 (5). However, articles on the incidence of IgG4-RD remain rare. In 2016, a national epidemiological survey was performed in Japan. The prevalence of AIP was 10.1/100,000 individuals, which more than doubled in 2011 (3).

A retrospective analysis of 103 patients previously diagnosed with an inflammatory pseudotumor was recently performed (12). Immunohistochemical analysis of $\operatorname{IgG}$ and IgG4 was performed on 16 biopsy samples and six cases of IgG4-ROD and 10 cases of orbital inflammatory pseudotumor were diagnosed. The aforementioned study (12) has also indicated that lesions in the lacrimal gland, IgG4-positive plasma-cell counts and ratios, as well as collagen fibrosis, are helpful for the diagnosis of IgG4-ROD in patients with orbital mass-like lesions. In addition, as an idiopathic inflammatory response, inflammatory pseudotumors are frequently accompanied by obvious pain. Tiegs-Heiden et al (13) reviewed the imaging features of 27 IgG4-ROD cases confirmed by biopsy. The vast majority of patients $(89 \%)$ had enlarged extraocular muscles. The external rectus was the most frequently involved muscle, while the inferior rectus and medial rectus were frequently involved in Graves' ophthalmopathy. A small number of patients may exhibit characteristics that differ from those described above, such as the case of the present study. If TSH receptor antibody is negative in a patient suspected of having Graves' orbital lesions with normal thyroid function, the presence of IgG4-ROD must be considered. In conclusion, when orbital masses occur, in addition to basic examinations, the state of the whole body should be assessed and biopsy tissues should be stained with IgG and IgG4 to exclude IgG4-ROD.

Painless eyelid swelling, mild exophthalmos and diplopia are common symptoms of IgG4-ROD. Due to the rare involvement of the optic nerve, visual impairment is an uncommon symptom $(14,15)$. In the reported cases, the symptoms of visual impairment were mostly due to the oppression of the optic nerve by the surrounding mass (16-19), which was not observed in the present case. Zhang et al (20) reported on the case of a 79-year-old woman with skin changes, pancreatic tumors, lymphadenopathy an eyelid mass and interstitial pneumonia for $>30$ years. Rapid vision loss occurred in the left eye 2 months prior to admission. On T2-weighted MRI, left optic atrophy with focal hyperintense lesions was observed and the latency time of visually evoked potential was prolonged. In addition, the patient did not have any compressive mass around the optic nerve, similar to the patient of the present study, suggesting that there may be novel mechanisms of visual impairment in IgG4-ROD. Li et al (21) reviewed 225 patients diagnosed with IgG4-RD between January 2014 and December 2017. Among these patients, 26 with decreased vision underwent orbital MRI and optic nerve injury was detected in all of them prior to treatment. The most common causes of optic nerve injury in these patients with IgG-4-ROD were inflammation of the optic nerve sheath (12 patients), compression of extraocular muscles and a pseudotumor mass (14 patients), hypertrophic meningitis (2 patients) and pituitary involvement in the optic chiasm (1 patient), where a patient may simultaneously exhibit $\geq 2$ causes of optic nerve injury, which suggests that pathological changes in the optic nerve, meninges and pituitary glands should not be ignored when investigating the mechanisms of IgG4-ROD.

In a large multicenter study by Yamada et al (22), the clinical and laboratory parameter characteristics of 334 patients with IgG4-RD were analyzed. A total of 205 patients were male and the average age at diagnosis was 65 years. The average number of organs affected at diagnosis was 3.2. The most frequently involved organs were the salivary glands, followed by the lacrimal glands, lymph nodes, pancreas, retroperitoneal/peri-aorta area, kidneys and lungs. It is rare for a patient to have 10 organs involved (in the patient described here: The eyes, submandibular glands, sinuses, middle ears, bile ducts, liver, pancreas, prostate, skin and lymph nodes were involved). Due to the early stages of disease progression in certain organs, only enzymatic changes were present and no changes were observed on imaging. Among these changes, liver injury was severe. The albumin concentration was as low as $21.2 \mathrm{~g} / 1$ at diagnosis. Hypoalbuminemia mimicked cirrhosis but returned to normal after hormone treatment.

The reported treatment options for IgG4-RD include systemic glucocorticoids, immunosuppressive drugs, biological agents and surgical resection of affected tissues. Except for contraindications, glucocorticoids are recommended as first-line drugs for remission induction in all active or untreated patients with IgG4-RD (23). According to the international consensus on the treatment of autoimmune pancreatitis, the initial dose of prednisone should be $0.6-1.0 \mathrm{mg} / \mathrm{kg} / \mathrm{day}$ and the minimum dose $20 \mathrm{mg} / \mathrm{day}$. The therapeutic effect may be evaluated for the first time after 2 weeks. Hormone reduction may be considered after 2-4 weeks as follows: A reduction by $5-10 \mathrm{mg}$ /day every $1-2$ weeks and a reduction by $5 \mathrm{mg} / \mathrm{day}$ every 2 weeks after $20 \mathrm{mg}$ /day until drug withdrawal (24). Glucocorticoid retreatment is suitable for patients who relapse after successful remission. Following relapse, the continuous use of steroid-sparing agents should be considered during remission maintenance periods (23).

In conclusion, IgG4-RD should be considered a differential diagnosis for patients who suffer longstanding symptoms involving multiple organs. Steroid therapy should be considered the first choice of treatment. 


\section{Acknowledgements}

Not applicable.

\section{Funding}

No funding was received.

\section{Availability of data and materials}

The datasets used and/or analyzed during the current study are available from the corresponding author on reasonable request.

\section{Authors' contributions}

$\mathrm{XW}$ and XS analyzed the data, performed a literature review and were major contributors in writing the manuscript. DL and YL encountered and treated the patient and directed the writing of the article. RA and ZZ collected and confirmed all data for this patient. All authors confirm the authenticity of the raw data and have read and approved the final manuscript.

\section{Ethics approval and consent to participate}

Not applicable.

\section{Patient consent for publication}

Informed written consent was obtained from the patient for publication of this case report and accompanying images.

\section{Competing interests}

The authors declare that they have no competing interests.

\section{References}

1. Kubo K and Yamamoto K: IgG4-related disease. Int J Rheum Dis 19: 747-762, 2016.

2. Deshpande V, Zen Y, Chan JK, Yi EE, Sato Y, Yoshino T, Klöppel G, Heathcote JG, Khosroshahi A, Ferry JA, et al: Consensus statement on the pathology of IgG4-related disease. Mod Pathol 25: 1181-1192, 2012.

3. Masamune A, Kikuta K, Hamada S, Tsuji I, Takeyama Y, Shimosegawa T and Okazaki K; Collaborators: Nationwide epidemiological survey of autoimmune pancreatitis in Japan in 2016. J Gastroenterol 55: 462-470, 2020.

4. Mejico LJ: IgG4-related ophthalmic disease. Saudi J Ophthalmol 29: 53-56, 2015.

5. Umehara H, Okazaki K, Masaki Y, Kawano M, Yamamoto M, Saeki T, Matsui S, Yoshino T, Nakamura S, Kawa S, et al: Comprehensive diagnostic criteria for IgG4-related disease (IgG4-RD), 2011. Mod Rheumatol 22: 21-30, 2012.

6. Tokura Y, Yagi H, Yanaguchi H, Majima Y, Kasuya A, Ito T, Maekawa $\mathrm{M}$ and Hashizume $\mathrm{H}$ : IgG4-related skin disease. Br J Dermatol 171: 959-967, 2016.

7. Agaimy A and Ihrler S: Immunoglobulin G4 (IgG4)-related disease. A review of head and neck manifestations. Pathologe 35: 152-159, 2014 (In German).
8. Minaga K, Watanabe T, Chung $\mathrm{H}$ and Kudo M: Autoimmune hepatitis and IgG4-related disease. World J Gastroenterol 25: 2308-2314, 2019.

9. Hamano H, Kawa S, Horiuchi A, Unno H, Furuya N, Akamatsu T, Fukushima M, Nikaido T, Nakayama, Usuda N and Kiyosawa K: High serum IgG4 concentrations in patients with sclerosing pancreatitis. N Engl J Med 344: 732-738, 2001

10. Hamano H, Kawa S, Ochi Y, Unno H, Shiba N, Wajiki M, Nakazawa K, Shimojo H and Kiyosawa K: Hydronephrosis associated with retroperitoneal fibrosis and sclerosing pancreatitis. Lancet 359: 1403-1404, 2002.

11. Kamisawa T, Funata N, Hayashi Y, Eishi Y, Koike M, Tsuruta K, Okamoto A, Egawa $\mathrm{N}$ and Nakajima $\mathrm{H}$ : A new clinicopathological entity of IgG4-related autoimmune disease. J Gastroenterol 38: 982-984, 2003.

12. Min HK, Lee YS, Yang SW, Lee J, Kwok SK, Ju JH, Kim WU and Park SH: Clinical outcomes and pathological characteristics of immunoglobulin G4-related ophthalmic disease versus orbital inflammatory pseudotumor. Korean J Intern Med 34: 220-226, 2019.

13. Tiegs-Heiden CA, Eckel LJ, Hunt CH, Diehn FE, Schwartz KM, Kallmes DF, Salomão DR, Witzig TE and Garrity JA: Immunoglobulin G4-related disease of the orbit: Imaging features in 27 patients. Am J Neuroradiol 35: 1393-1397, 2014.

14. Andrew N, Kearney D and Selva D: IgG4-related orbital disease: A meta-analysis and review. Acta Ophthalmol 91: 694-700, 2013.

15. Kubota T and Moritani S: Orbital IgG4-related disease: Clinical features and diagnosis. ISRN Rheumatol 2012: 412896, 2012.

16. Takahashi Y, Kitamura A and Kakizaki H: Bilateral optic nerve involvement in immunoglobulin G4-related ophthalmic disease. J Neuroophthalmol 34: 16-19, 2014.

17. Takahira M, Ozawa Y, Kawano M,Zen Y, Hamaoka S, Yamada K and Sugiyama K: Clinical aspects of IgG4-related orbital inflammation in a case series of ocular adnexal lymphoproliferative disorders. Int J Rheumatol 2012: 635473, 2012.

18. Ramirez L, D'Auria A, Popalzai A and Sanossian N: Bilateral vision loss secondary to pachymeningitis in a patient with IgG4-related disease. Front Neurol 5: 192, 2014.

19. Sogabe Y, Ohshima K, Azumi A, Takahira M, Kase S, Tsuji H, Yoshikawa $\mathrm{H}$ and Nakamura T: Location and frequency of lesions in patients with IgG4-related ophthalmic diseases. Graefes Arch Clin Exp Ophthalmol 252: 531-538, 2014.

20. Zhang W, Luo J and Jiao J: Optic nerve involvement in immunoglobulin G4-related disease: A case report. Exp Ther Med 12: 111-114, 2016

21. Li J, Zhang Y, Zhou H, Wang L, Wang Z and Li H: Magnetic resonance imaging indicator of the causes of optic neuropathy in IgG4-related ophthalmic disease. BMC Med Imaging 19: 49, 2019.

22. Yamada K, Yamamoto M, Saeki T, Mizushima I, Matsui S, Fujisawa Y, Hara S, Takahashi H, Nomura H, Kawa S and Kawano M: New clues to the nature of immunoglobulin G4-related disease: A retrospective Japanese multicenter study of baseline clinical features of 334 cases. Arthritis Res Ther 19: $262,2017$.

23. Khosroshahi A, Wallace ZS, Crowe JL, Akamizu T, Azumi A, Carruthers MN, Chari ST, Della-Torre E, Frulloni L, Goto $\mathrm{H}$, et al: International consensus guidance statement on the management and treatment of IgG4-related disease. Arthritis Rheumatol 67: 1688-1699, 2015.

24. Okazaki K, Chari ST, Frulloni L, Lerch MM, Kamisawa T, Kawa S, Kim MH, Lévy P, Masamune A, Webster G and Shimosegawa T: International consensus for the treatment of autoimmune pancreatitis. Pancreatology 17: 1-6, 2017

This work is licensed under a Creative Commons Attribution-NonCommercial-NoDerivatives 4.0 International (CC BY-NC-ND 4.0) License. 